\title{
PRODUÇÃO DE FERRITA DE COBALTO POR COPRECIPITAÇÃO EM MEIO OXALATO
}

\author{
I.A.F. MEDEIROS ${ }^{1}$, D.C. ANDRADE ${ }^{1}$, A.L. LOPES-MORIYAMA', CH. LEROUX², \\ C.P. de SOUZA ${ }^{1}$ \\ ${ }^{1}$ Universidade Federal de Santa Catarina, Departamento de Engenharia Química \\ ${ }^{2}$ Université de Toulon, IM2NP, La Garde, France \\ E-mail para contato: indiraafm@gmail.com
}

\begin{abstract}
RESUMO - O desenvolvimento de cerâmicas surgiu como um dos campos mais promissores da área de novos materiais, tanto sob o ponto de vista experimental quanto teórico. Dentre esses materiais, as nanopartículas de ferrita de cobalto têm sido utilizadas como material de base em uma grande variedade de aplicações, tais como: sensores de gás, dispositivos de microondas, e catalisadores em reações de oxidação. Neste contexto, o presente trabalho propõe a obtenção de ferrita de cobalto em reator em batelada pelo método de coprecipitação em meio oxalato, e a análise da influência dos parâmetros de síntese na pureza dos pós e no tamanho de cristalito. Os experimentos foram realizados de acordo com planejamento experimental $2^{3}$. As variáveis independentes são o pH do meio reacional, a temperatura e o tempo de calcinação. Os pós foram caracterizados por DRX, EDS e FRX. Verificou-se que o pH é o fator de maior influência na pureza do material. Foi possível gerar modelo estatístico adequado aos resultados.
\end{abstract}

\section{INTRODUÇÃO}

A cada dia mais a tecnologia vem sendo desenvolvida para a melhoria da vida humana. Para isso, as descobertas minuciosas no âmbito atômico consistem em um diferencial inovador e revolucionário. A área da nanotecnologia se mostra como uma forma de criar, manipular e explorar materiais em escalas nanométricas, permitindo o aprimoramento e controle das propriedades dos mesmos. Sua aplicabilidade vai desde o setor médico até o eletrônico e químico (Durán et al, 2006). Dentre o vasto mundo da nanotecnologia, as nanopartículas magnéticas de ferrita de cobalto $\left(\mathrm{CoFe}_{2} \mathrm{O}_{4}\right)$ vêm sendo aplicadas, como, por exemplo, no campo de reações catalíticas. (Feng et al, 2013; Zhao e Ma, 2010; Tong, et al, 2009).

O material em estudo deste trabalho, ferrita de cobalto, é um composto cerâmico com baixo custo de obtenção, alta resistência mecânica e estabilidade química. À temperatura ambiente apresenta estrutura cristalina cúbica do tipo espinélio inverso, que possibilita a este material propriedades magnéticas diferenciadas (Turtelli et al., 2012; Sajjia et al, 2010; Santos, 2008). Contudo, tais características e propriedades dependem do tamanho de cristalito e da uniformidade de suas partículas, as quais são suscetíveis aos parâmetros e métodos de preparação adotados. Desta forma, o presente trabalho tem como objetivo a obtenção de ferrita de cobalto nanoestruturada em 
reator em batelada através do método de coprecipitação via oxalato, assim como, o estudo da influência dos parâmetros de síntese na pureza dos pós e no tamanho de cristalito. Para isso, os experimentos foram realizados de acordo com planejamento experimental $2^{3}$ baseado nos seguintes parâmetros: $\mathrm{pH}$ do meio reacional, temperatura e tempo de calcinação Foram feitas três repetições no ponto central, totalizando onze sínteses. Os pós obtidos foram caracterizados por difração de raios-X (DRX), espectroscopia de energia dispersiva (EDS) e fluorescência de raios-X (FRX).

\section{METODOLOGIA}

\subsection{Síntese}

A produção de ferrita de cobalto foi realizada por meio da coprecipitação via oxalato. As matérias-primas utilizadas foram Nitrato de Cobalto II hexahidratado $\left(\mathrm{Co}\left(\mathrm{NO}_{3}\right)_{2} \cdot 6 \mathrm{H}_{2} \mathrm{O}\right)$, Nitrato de Ferro (III) nonahidratado $\left(\mathrm{Fe}\left(\mathrm{NO}_{3}\right)_{3} \cdot 9 \mathrm{H}_{2} \mathrm{O}\right)$, Oxalato de Amônio $\left(\mathrm{C}_{2} \mathrm{H}_{8} \mathrm{~N}_{2} \cdot \mathrm{H}_{2} \mathrm{O}\right)$, Ácido Oxálico $\left(\mathrm{C}_{2} \mathrm{H}_{2} \mathrm{O}_{4} .2 \mathrm{H}_{2} \mathrm{O}\right)$ e Trietilamina. Inicialmente, foram preparadas duas soluções homogêneas a partir dos nitratos contendo os cátions de interesse, e do oxalato de amônio e ácido oxálico. Em seguida, essas soluções foram misturadas simultaneamente sob controle de $\mathrm{pH}$ com a adição de trietilamina em um reator de mistura em batelada, formando um precipitado. Esse precipitado foi lavado e em seguida secado a temperatura de $80^{\circ} \mathrm{C}$ por 10 horas. O material seco foi, então, calcinado com taxa de aquecimento de $1^{\circ} \mathrm{C} / \mathrm{min}$ de acordo com os parâmetros mostrados na Tabela 1 .

\subsection{Planejamento experimental}

Para auxiliar na quantificação da influência dos parâmetros de síntese na pureza e no tamanho do cristalito de $\mathrm{CoFe}_{2} \mathrm{O}_{4}$, foi realizado um planejamento experimental fatorial do tipo $2^{3}$ com três repetições no ponto central. O modelo desenvolvido é baseado em interações de primeira ordem, que, genericamente, são representadas pela Equação 1.

$$
y=b_{0}+\sum_{i=1}^{k} b_{i} x_{i}+\sum_{i=1}^{k} \sum_{j=1}^{k} b_{i, j} x_{i} x_{j}+\varepsilon
$$

Onde o coeficiente $y$ representa a variável dependente; $b_{0} \mathrm{o}$ valor médio de todas as respostas obtidas; $b_{i}$ o coeficiente linear; $x$ as variáveis independentes; $b_{i, j}$ os coeficientes para as interações entre as variáveis independentes, e $\varepsilon$ o erro aleatório associado ao modelo.

Tabela 3.3 - Níveis de fatores das variáveis independentes.

\begin{tabular}{|c|c|c|c|}
\hline Fatores & \multicolumn{3}{|c|}{ Níveis } \\
\hline Codificado & -1 & 0 & 1 \\
\hline$x_{1}-\mathrm{pH}$ & 8 & 9 & 10 \\
\hline$x_{2}-$ Temperatura $\left({ }^{\circ} \mathrm{C}\right)$ & 600 & 800 & 1000 \\
\hline$x_{3}-$ Tempo $(\mathrm{h})$ & 2 & 4 & 6 \\
\hline
\end{tabular}




\subsection{Caracterização}

Os pós cerâmicos sintetizados para este trabalho foram caracterizados por Difração de Raios-X (DRX), utilizando difratômetro de Raios-X modelo DRX - 6000 da SHIMADZU com radiação de $\mathrm{Cu}-\mathrm{K} \alpha$. Os difratogramas foram obtidos na faixa de $2 \theta$ de $10^{\circ}$ a $75^{\circ} \mathrm{com}$ taxa de varredura de $1^{\circ} / \mathrm{min}$ em passos de $0,02^{\circ}$. Por meio dos difratogramas foi possível analisar a pureza da fase e estimar o tamanho de cristalito a partir de um perfil gerado pelo software PANalytical X'Pert PRO@. Foram utilizadas as equações de Halder-Wagner-Langford (HWL) para a determinação do tamanho de cristalito. Um espectroscópio de energia dispersiva TM-3000 foi utilizado para realizar análise química das amostras. Para confirmar os resultados obtidos, foi realizada fluorescência de Raios-X no equipamento do tipo EDX-720 da marca Shimadzu.

\section{RESULTADOS E DISCUSSÕES}

Na Figura 1 são apresentados os difratogramas de raios-X obtidos para as amostras produzidas.

a)

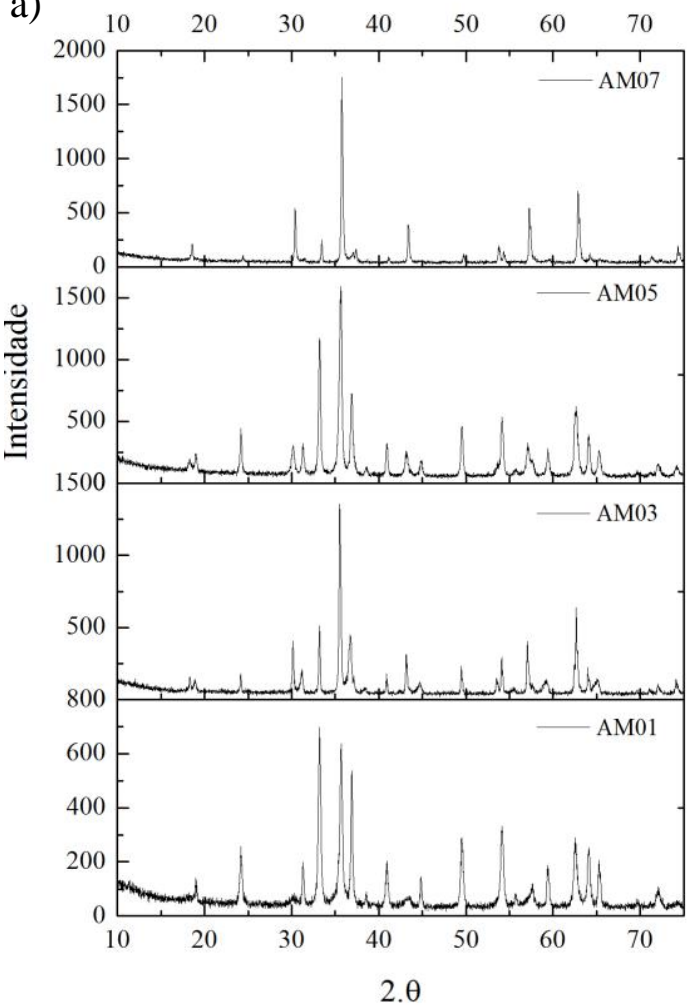

b)

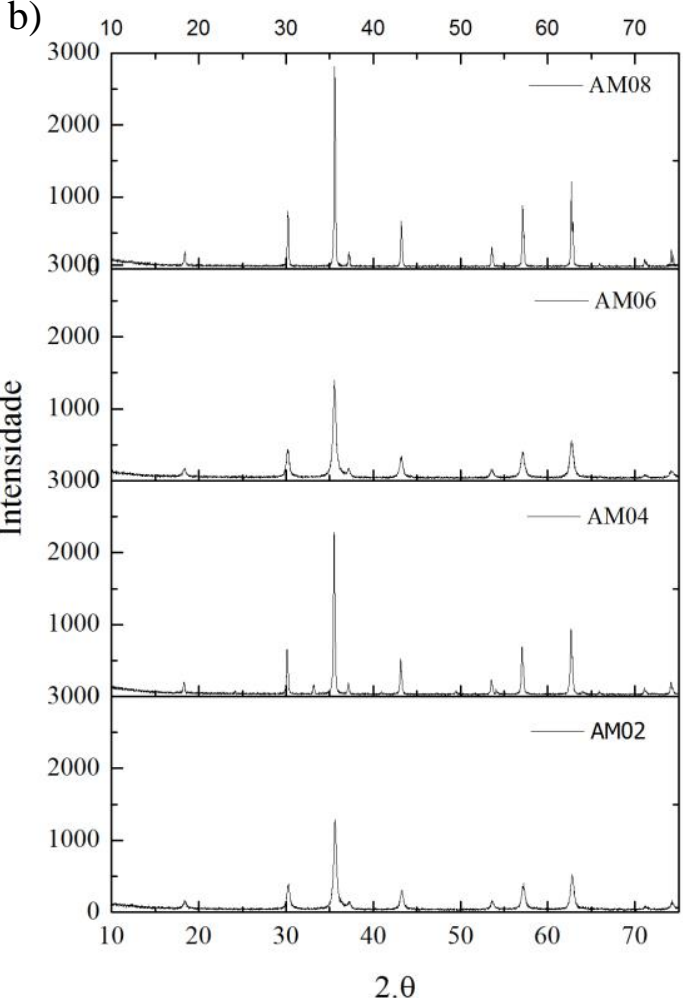

Figura 1: Difratograma de raios-X: (a) $\mathrm{CoFe}_{2} \mathrm{O}_{4}$ sintetizada com $\mathrm{pH}$ igual a 8. (b) $\mathrm{CoFe}_{2} \mathrm{O}_{4}$ sintetizada com $\mathrm{pH}$ igual a 10.

Avaliando a Figura 1b, pode-se afirmar que foi possível obter $\mathrm{CoFe}_{2} \mathrm{O}_{4}$ com estrutura cúbica do tipo espinélio a partir de $600^{\circ} \mathrm{C}$ (AM02) para reações cujo pH do meio reacional foi igual a 10 , já que não são observados picos adicionais à carta padrão ICSD 109045. Nota-se, também, que o aumento 
da temperatura de calcinação proporciona a diminuição dos ruídos e o aumento da intensidade dos picos, o que está relacionado com o crescimento do tamanho de cristalito e o aumento da cristalinidade das amostras. A partir da Figura 1a verifica-se que não foi possível obter $\mathrm{CoFe}_{2} \mathrm{O}_{4}$ para as reações com pH do meio reacional igual a 8, já que são constatados picos adicionais à carta padrão ICSD 109045. Os picos adicionais são identificados nas fases de hematita (ICSD 082903) e óxido de cobalto (ICSD 063165).

Na Tabela 2 é apresentado o tamanho de cristalito estimado após o tratamento da identidade cristalográfica do material, assim como a pureza do pós obtida através de análise de DRX, EDS e FRX.

Tabela 2 - Tamanho de cristalito e pureza das amostras produzidas.

\begin{tabular}{|c|c|c|c|c|}
\hline $\mathrm{AM}$ & $\mathrm{TC}(\mathrm{nm})$ & $\% \mathrm{CoFe}_{2} \mathrm{O}_{4}-\mathrm{DRX}$ & $\% \mathrm{CoFe}_{2} \mathrm{O}_{4}-\mathrm{EDS}$ & $\% \mathrm{CoFe}_{2} \mathrm{O}_{4}-\mathrm{FRX}$ \\
\hline 01 & 37 & 34 & 98 & 67 \\
\hline 02 & 32 & 100 & 99 & 97 \\
\hline 03 & 185 & 56 & 83 & 99 \\
\hline 04 & 179 & 95 & 98 & 85 \\
\hline 05 & 33 & 44 & 86 & 75 \\
\hline 06 & 32 & 100 & 98 & 95 \\
\hline 07 & 147 & 89 & 67 & 82 \\
\hline 08 & 326 & 100 & 98 & 98 \\
\hline 10 & 75 & 34 & 73 & 95 \\
\hline 11 & 98 & 52 & 64 & 96 \\
\hline 12 & 70 & 39 & 58 & 100 \\
\hline
\end{tabular}

Analisando a Tabela 2, verifica-se que o percentual de ferrita de cobalto resultante no pó obtido para cada condição do planejamento experimental apresenta valores distintos entre as técnicas de caracterização estudadas. Isso ocorre porque as impurezas encontradas nas amostras apresentam os mesmos elementos da ferrita de cobalto $\left(\mathrm{Fe}_{2} \mathrm{O}_{3}\right.$ e/ou $\left.\mathrm{Co}_{3} \mathrm{O}_{4}\right)$, assim, não é possível determinar através das técnicas de EDS e FRX a real composição deste pó, já que a primeira técnica é caracterizada pela análise pontual de uma determinada região escolhida aleatoriamente, podendo, assim, existir regiões com maior ou menor concentração da impureza, e a segunda através da média das concentrações dos elementos existentes. Desta forma, a avaliação estatística dos parâmetros de síntese na pureza do pó foi realizada a partir dos dados de composição resultantes da análise de DRX, a qual é calculada a partir da quantificação da intensidade dos picos principais de cada fase presente no difratograma.

Nas Figuras 2a e 2b são apresentados os diagramas de pareto para as variáveis dependentes tamanho de cristalito (Figura 2a) e pureza (Figura 2b). Na Figura 2a, verifica-se que todos os efeitos com magnitude superior a 4,30 (p=0,05) apresentam significância estatística no tamanho do cristalito. A temperatura foi o parâmetro com maior influência sobre a resposta, e sua elevação proporciona o aumento do tamanho de cristalito. Além disso, constata-se que apesar dos fatores $\mathrm{pH}$ e tempo de 
calcinação não apresentarem efeito pertinente, a sua combinação é significativa e seu aumento gera um aumento proporcional na variável resposta. Uma possível explicação da dependência do tamanho de cristalito com o aumento da temperatura e do tempo de reação está relacionada ao aumento da temperatura proporcionar um aumento da energia do sistema e consequentemente um aumento da entropia, então para minimizar essa energia os cristais coalescem, formando cristalitos maiores. Já o aumento do tempo de calcinação promove um maior tempo de contato entre esses cristais, favorecendo, também, a coalescência dos mesmos, aumentando, assim, o tamanho do cristalito.

Contudo, com relação à pureza dos pós obtidos, constata-se que o $\mathrm{pH}$ do meio reacional é o único fator que apresenta efeito significante, contribuindo para a obtenção de ferrita de cobalto com maior pureza, conforme o apresentado na Figura 2b. Acredita-se que este fenômeno pode ser explicado em função da solubilização do cobalto na fase líquida quando o $\mathrm{pH}$ do meio reacional é inferior a 10, proporcionando a formação de hematita e óxido de cobalto como impureza na ferrita de cobalto.

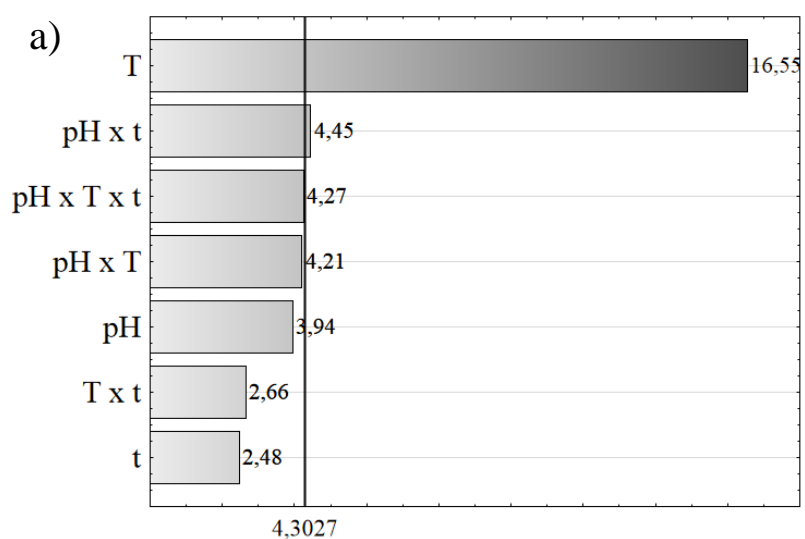

Efeitos Padronizados (Valor Absoluto)

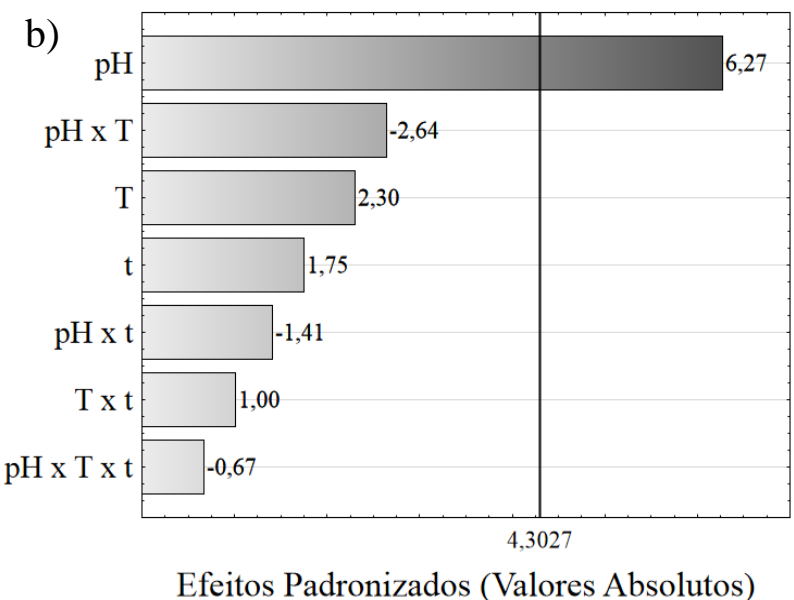

Efeitos Padronizados (Valores Absolutos)

Figura 2 - Diagrama Pareto: (a) Tamanho de cristalito. (b) Pureza.

As influências desses fatores no tamanho de cristalito e na pureza podem ser melhor visualizadas na superfície de resposta. A Figura 3a mostra a influência da temperatura e do tempo de calcinação no tamanho de cristalito. Este gráfico revela que o efeito da temperatura de calcinação no tamanho de cristalito foi mais evidente que o do tempo de calcinação. Além disso, contata-se que cristalitos menores são obtidos nas condições mínimas de temperatura e que a altas temperaturas o aumento do tempo de calcinação contribui significativamente para o crescimento do cristalito. De acordo a Figura 3b, o pH do meio reacional apresenta maior efeito na pureza do pó que a temperatura de calcinação, e seu aumento gera produtos com maior pureza. 

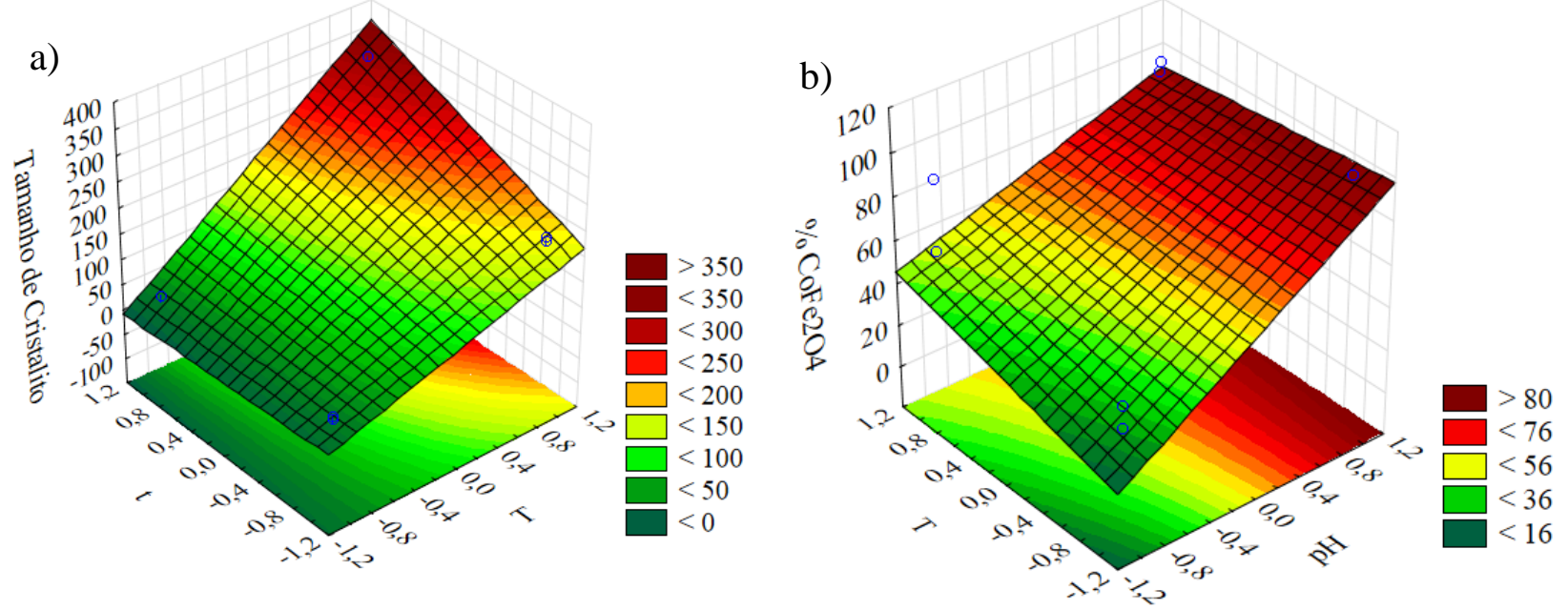

Figura 3 - Superfície de resposta: (a) Tamanho de cristalito para as amostras obtidas com pH do meio reacional igual a 10. (b) Composição para as amostras calcinadas por 2 horas.

A partir da regressão das superfícies de respostas foi possível obter um modelo com significância estatística de $95 \%$ para o tamanho do cristalito e pureza. As Equações 1 e 2 apresentam o modelo codificado dos efeitos significativos para essas variáveis.

$$
\begin{aligned}
& T C=81,60+59,42 \cdot x_{2}-31,98 \cdot x_{1} \cdot x_{2} \\
& \% \mathrm{CoFe}_{2} \mathrm{O}_{4}=67,66+42,75 \cdot x_{1}
\end{aligned}
$$

Onde, $T C$ é o tamanho de cristalito, $\% \mathrm{CoFe}_{2} \mathrm{O}_{4}$ é a pureza da ferrita de cobalto, $x_{1}$ representa o pH do meio reacional e $x_{2}$ a temperatura de calcinação.

Objetivando comprovar o bom ajuste do modelo, assim como a sua capacidade de predição dos dados experimentais, foram realizados o teste de significância do modelos, e o teste da falta de ajuste. Os resultados obtidos para essa análise são observados na Tabela 2.

Tabela 2 - Análise de variância - ANOVA - para as duas respostas.

\begin{tabular}{|c|c|c|c|c|c|c|c|c|}
\hline & \multicolumn{4}{|c|}{ Tamanho de cristalito } & \multicolumn{4}{c|}{ Pureza } \\
\hline & SS & DF & MS & F & SS & DF & MS & F \\
\hline Regressão & 66215,07 & 2 & 33107,53 & 11,33 & 3655,80 & 1 & 3655,80 & 12,58 \\
\hline Resíduo & 14604,12 & 5 & 2920,82 & - & 1744,03 & 6 & 290,67 & - \\
\hline Falta de Ajuste & 3484,35 & 1 & 3484,35 & 7,72 & 2817,54 & 1 & 2817,54 & 30,27 \\
\hline Erro Puro & 451,00 & 2 & 225,5 & - & 186,19 & 2 & 93,09 & - \\
\hline Total & 84754,54 & 10 & 8475,45 & & 5399,83 & 10 & 539,98 & \\
\hline
\end{tabular}




\section{9 a 22 de outubro de 2014 \\ Florianópolis/SC}

A análise da ANOVA mostra que ambos os modelos apresentam significância estatística com 95\% de confiança, uma vez que os valores de F são maiores que os valores tabelados da distribuição de Fisher: 1,92 vezes para o modelo obtido para o tamanho de cristalito $\left(F_{\text {Fisher }}=5,99\right.$ ) e 2,10 vezes para o obtido para a pureza do pó $\left(\mathrm{F}_{\text {Fisher }}=5,79\right)$. No entanto, o teste da falta de ajuste mostra que apenas o modelo obtido para o tamanho de cristalito é preditivo, já que apenas para esse modelo o valor de $\mathrm{F}$ foi inferior ao da distribuição de Fisher: 0,41 vezes $\left(\mathrm{F}_{\text {Fisher }}=18,51\right)$. Para o modelo que descreve a pureza do pó, o valor de $\mathrm{F}$ é superior ao da distribuição de Fisher:1,64 vezes maior $\left(\mathrm{F}_{\text {Fisher }}\right.$ = 18,51). Este comportamento é resultante da ocorrência da fase de hematita na amostra AM04 apresentado na Figura 1. Acredita-se que a ocorrência dessa fase é consequência da sensibilidade do sistema a variações do $\mathrm{pH}$ do meio durante a síntese. Durante esta reação foi registrado uma rápida queda do pH do meio reacional de 10 para 9. Entretanto, essa variação não apresentou efeito nos resultados obtidos para a variável tamanho de cristalito, pois, como pode ser observado na Figura $2^{\mathrm{a}}$, o $\mathrm{pH}$ do meio reacional não apresenta efeito pertinente sobre esse variável.

Com o objetivo de verificar a capacidade de predição dos modelos, foi construído o gráfico dos valores preditos e valores observados. Os mesmos são mostrados na Figura 3. Os valores ideais são representados por uma reta, enquanto os valores observados experimentalmente são representados por pontos. Avaliando a Figura 3a se constata que os valores experimentais aproximam-se da linha de valores ideais, apresentando um pequeno desvio em apenas duas amostras. Isto confirma a adequação do modelo obtido para o tamanho de cristalito. Por outro lado, analisando na Figura 3 b verifica-se que mais da metade dos valores experimentais encontram-se afastados da linha de idealidade, indicando a inadequação do modelo obtido para a pureza do pó.
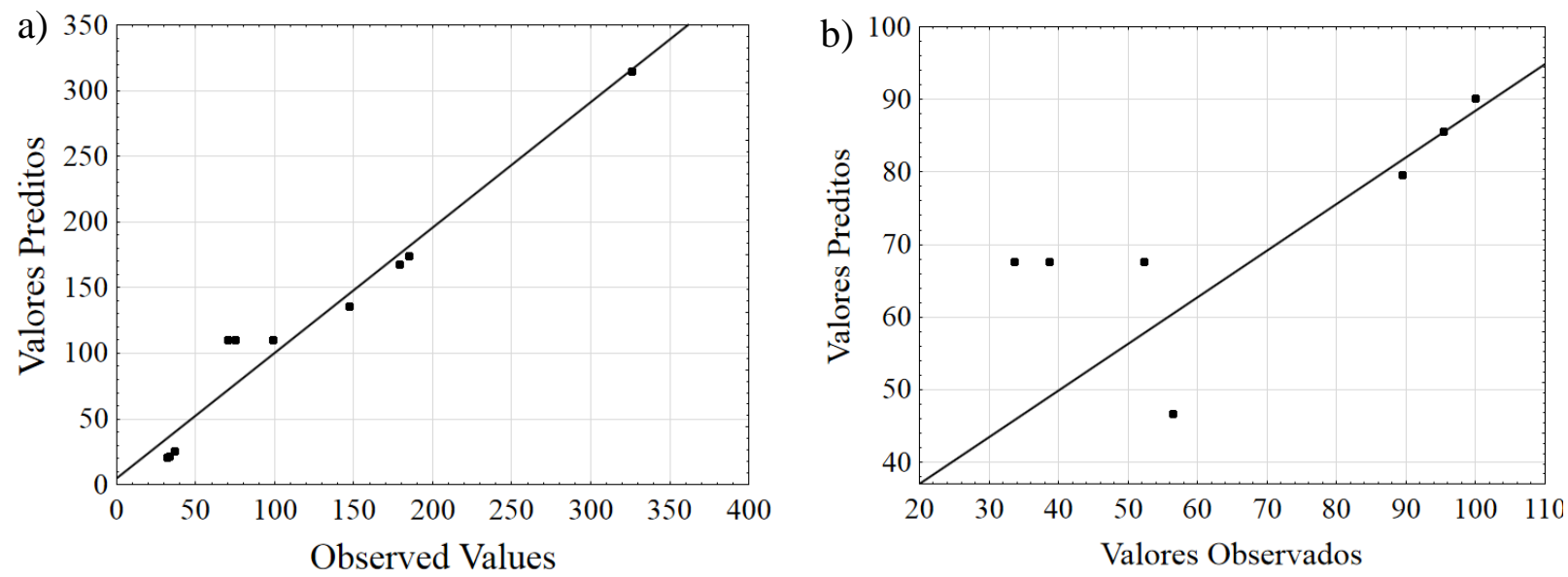

Figura 3 - Valores preditos versus valores observados para: (a) Tamanho de cristalito. (b) Pureza.

\section{CONCLUSÕES}

Nesse estudo foi possível obter nanocristais de $\mathrm{CoFe}_{2} \mathrm{O}_{4}$ puros a partir do método de coprecipitação via oxalato a temperatura de $600^{\circ} \mathrm{C}$ com tamanho médio de cristalito igual a $32 \mathrm{~nm}$. A partir do planejamento fatorial foi possível obter modelo estatístico para ambas as variáveis dependentes estudadas (tamanho de cristalito e pureza). O modelo desenvolvido para a pureza do pó 
se mostrou não ajustado aos dados experimentais. Mais experimentos são necessários para a adequação do mesmo. Foi observado que a temperatura de calcinação é o principal parâmetro para o controle do tamanho do cristalito, e o $\mathrm{pH}$ do meio reacional para a pureza do produto final. Todavia, apesar do pH e do tempo de calcinação não influenciarem no tamanho de cristalito quando analisado separadamente, a combinação desses fatores proporciona o crescimento do tamanho do cristalito.

\section{REFERÊNCIAS}

DURÁN, N.; MATTOSO, L. H. C.; MORAIS, P. C. Nanotecnologia: Intrudução, preparação e caracterização de nanomateriais e exemplos de aplicações. Editora Artliber, 2006.

FENG, X.; MAO, G. Y.; BU, F. X.; CHENG , X. L.; JIANG, D.M.; JIANG, J. S. Controlled synthesis of monodisperse $\mathrm{CoFe} 2 \mathrm{O} 4$ nanoparticles by the phase transfer method and their catalytic activity on methylene blue discoloration with H2O2. J. of Magnetism and Magnetic Materials. v343, p126-132, 2013.

SAJJIA, M.; OUBAHA, M.; PRESCOTT, T.; OLABIA, A. G. Development of cobalt ferrite powder preparation employing the sol-gel technique and its structural characterization. Journal of Alloys and Componds. v506, p400-406, 2010.

SANTOS, C. M. B. Efeito da variação do processo de síntese nas propriedades estruturais de nanopartículas de CoFe2O4. 2008. 102f. Dissertação (Mestrado em Física) - Centro de Ciências, Departamento de Física, Programa de Pós-Graduação em Física, Universidade de Brasília, Brasília.

TONG, J.; BO, L.; LI, Z.; LEI, Z.; XIA, C. Magnetic CoFe2O4 nanocrystal: A novel and efficient heterogeneous catalystfor aerobic oxidation of cyclohexane. Journal of Molecular Catalysis A: Chemical. v307, p58-63, 2009.

TURTELLI, R. S.; ATIF, M. ; MEHMOOD, N. ; KUBEL, F. ; BIERNACKA, K. ; LINERT, W. ; GROSSINGER, R. ; KAPUSTA, Cz. ; SIKORA, M. Interplay between the cation distribution and production methods in cobalt ferrite. Materials Chemistry and Physics. v.133, p.832-838, 2012.

ZHAO, S. ; MA, D. Preparation of CoFe2O4 Nanocrystallites by Solvothermal Process and Its Catalytic Activity on the Thermal Decomposition of Ammonium Perchlorate. Journal of Nanomaterials, v.2010, p48, 2010. 